\title{
Hemodynamic Alteration in the Liver in Acute Hepatitis: A Quantitative Evaluation Using Computed Tomographic Perfusion
}

\author{
AKIHIRO NISHIE ${ }^{1,2}$, YASUHIRO USHIJIMA ${ }^{3}$, YUKIHISA TAKAYAMA ${ }^{3}$, NOBUHIRO FUJITA ${ }^{3}$, YUICHIRO KUBO $^{3}$, \\ KEISUKE ISHIMATSU ${ }^{3}$, DAISUKE TSURUMARU ${ }^{3}$, MOTOYUKI KOHJIMA ${ }^{4}$ and KOUSEI ISHIGAMI ${ }^{3}$ \\ ${ }^{1}$ Department of Radiology, Graduate School of Medical Science, University of the Ryukyus, Okinawa, Japan; \\ ${ }^{2}$ Department of Advanced Imaging and Interventional Radiology, \\ Graduate School of Medical Sciences, Kyushu University, Fukuoka, Japan; \\ ${ }^{3}$ Department of Clinical Radiology, Graduate School of Medical Sciences, Kyushu University, Fukuoka, Japan; \\ ${ }^{4}$ Department of Medicine and Bioregulatory Science, \\ Graduate School of Medical Sciences, Kyushu University, Fukuoka, Japan
}

\begin{abstract}
Background/Aim: We aimed to elucidate the hemodynamic alterations in the liver of patients with acute hepatitis (AH) using computed tomography perfusion imaging. Patients and Methods: For 14 patients with AH and nine patients with no disease (ND group), we compared the mean arterial blood flow $(A F)$, portal blood flow $(P F)$ and perfusion index (\%) $[P I=A F /(A F+P F) \times 100]$ of the right and left liver lobes and investigated their relationship with clinical factors. Results: The mean PI of the right lobe in the AH group (30.5 $\pm 10.0 \%)$ was significantly higher than that in the ND group $(20.8 \pm 9.7 \%)(p=0.031)$. For all patients of the $A H$ and ND groups, the PI of the right lobe was increased as the prothrombin time decreased $(R=-0.56$, $p=0.006)$ and as the prothrombin time-international normalized ratio increased $(R=0.48, p=0.02)$. Conclusion: The PI of the right liver lobe may increase in AH and may be a predictive parameter for the severity of hepatic failure.
\end{abstract}

Although it is accepted that acute hepatitis $(\mathrm{AH})$ is self-limiting in most patients, it can progress to acute liver failure in some and further progress to fulminant liver failure with a high mortality rate (1). Early treatment for acute or fulminant liver failure can effectively reduce the mortality but this remains

Correspondence to: Akihiro Nishie, MD, Department of Radiology, Graduate School of Medical Science, University of the Ryukyus, 207, Uehara, Nishihara-cho, Okinawa, 903-0215, Japan. Tel.: +81 988951162, Fax: +81988951420, e-mail: nishie_a@med.uryukyu.ac.jp

Key Words: Acute hepatitis, CT perfusion, hemodynamic alteration. This article is freely accessible online. challenging, one of the reasons being that there are no reliable and widely accepted procedures for distinguishing between self-limiting and fatal disease. Another reason is the lack of a sufficient understanding of the mechanisms of fulminant liver failure. Only the mechanism that underlies fulminant hepatic failure originating from acetaminophen has been established (2). Primarily through the action of cytochrome P-450 2E1, acetaminophen is metabolized to $N$-acetyl- $p$-benzo-quinone imine, which leads to hepatocyte death by its combination with DNA. Another assumed mechanism of fulminant hepatic failure is excessive reactions by cellular immunity (3). In cases of hepatitis virus-mediated acute hepatic failure, cytotoxic Tlymphocytes may destroy hepatocytes by recognizing the viral antigen on the surface of the cell membrane. It was proposed that a microcirculation disorder might be associated with the development of fulminant hepatic failure, based on the accumulation of fibrin in hepatic sinusoids (4). A previous report also suggested that activated macrophages in the liver might play a key role in the development of fulminant hepatic failure (5). Hypercytokinemia due to an abnormal activation of macrophages may cause microvascular disorder in the liver. Therefore, if hemodynamic alterations in the liver could be objectively evaluated, this knowledge would contribute to the elucidation of underlying mechanisms, development of therapeutic strategies, and to personalized medicine for acute hepatic failure.

Computed tomographic (CT) perfusion imaging is well documented as a valid quantitative method for identifying liver hemodynamic properties in several hepatic entities, and it has already been clinically applied (6-10). The CT perfusion imaging procedure can compensate for the limitations in the use of ultrasound, which reveals only blood velocity. The dual-input maximum-slope method was also developed for 
liver perfusion analyses, and it has enabled the quantification of arterial and portal hemodynamics (6-10). We hypothesized that this method might be the optimal choice for evaluating hemodynamic alterations in $\mathrm{AH}$ in detail.

We thus conducted the present study to elucidate hemodynamic alterations of the liver in $\mathrm{AH}$ by using $\mathrm{CT}$ perfusion imaging and to investigate the relationship between these alterations and the severity of hepatic failure.

\section{Patients and Methods}

Study population. From September 2012 to December 2015 at our Institution, 17 patients with $\mathrm{AH}$ underwent $\mathrm{CT}$ perfusion imaging on their admission day for the evaluation of the morphology and hemodynamics of the liver. Two of the patients were excluded from the present analyses due to poor image quality, and another was excluded due to a past history of treatment for acute liver failure. A total of 14 patients were therefore enrolled in this study (AH group) and comprised nine men and five women (age range $=18-71$ years, mean age $=49$ years). The underlying disease was hepatitis A or B virus in five patients, drug-induced in four, alcohol abuse in two, autoimmune hepatitis in two, and Wilson disease in one. Of the 14 patients, nine patients were diagnosed with acute hepatic failure and three were diagnosed with fulminant hepatic failure.

Acute hepatic failure was defined as a prothrombin time (PT) $\leq 40 \%$ or a PT-international normalized ratio (INR) $>1.5$. Fulminant hepatic failure was defined as hepatic encephalopathy $>$ grade II in addition to a PT $\leq 40 \%$ or a PT-INR $>1.5$. In addition to PT and PT-INR, the patient's platelet count and levels of alanine aminotransferase (ALT), lactate dehydrogenase (LDH), fibrinogen/ fibrin degradation products, D-dimer, and ferritin measured on the admission day were also recorded. The ratio of ALT to LDH (ALT/LDH) was also calculated.

The treatments were plasma exchange in 10, arterial steroid injection therapy for the liver in six, steroid pulse therapy in four, and liver transplantation in two. At time of this writing, one patient has died and the others remain alive.

We also analyzed the cases of another 10 patients with gastric cancer who underwent CT perfusion imaging at our Institution for the evaluation of the morphology and hemodynamics of a tumor during the period from April 2014 to June 2014. One of these patients was excluded due to the presence of liver metastases, and thus nine patients were enrolled. This group of patients with no disease (ND group) comprised seven men and two women (age range $=55-77$ years, mean age $=67$ years). None of these patients had underlying liver disease nor a history of chemotherapy. The liver function of each patient was within the normal range. These patients' PT, PT-INR, platelet count, ALT and LDH measured on the admission day were recorded. ALT/LDH was also calculated.

Institutional Review Board approval for this retrospective study was obtained (no. 26-251). All of the participants gave their written informed consent prior to their CT scans.

CT imaging. All examinations were performed with a 320-detecter row CT (Aquilion ONE; Toshiba Medical Systems, Tokyo, Japan). The scan range was $16 \mathrm{~cm}$, and as much of the liver as possible was included in the scans. Forty milliliters of iodinated contrast medium (Omnipaque 350; Daiichi-Sankyo, Tokyo, Japan) was administered at a rate of $5 \mathrm{ml} / \mathrm{s}$ with a power injector, followed by $20 \mathrm{ml}$ of saline chaser. Dynamic scans were performed 4-64 s after the injection of contrast material under respiration. Images were acquired with the following parameters: 0.5 -mm thickness, 320 slices, $512 \times 512$ matrices, $100 \mathrm{kV}$, and $120 \mathrm{~mA}$. The first 10 scans were performed every $2 \mathrm{~s}$. The next $10 \mathrm{scans}$ were performed every $3 \mathrm{~s}$, and the last three scans were performed every $4 \mathrm{~s}$. The CT exposure dose index volume was $64.4 \mathrm{mGy}$, the dose-length product was 1,030 $\mathrm{mGy} \times \mathrm{cm}$, and the effective dose was $15.5 \mathrm{mSv}$.

A portal venous phase abdominal-to-pelvic CT scan was acquired $60 \mathrm{~s}$ after the additional injection of $60 \mathrm{ml}$ of contrast material at a rate of $2 \mathrm{ml} / \mathrm{s}$ to evaluate the patency of vessels and the morphology of the liver and other organs. The imaging acquisition parameters were as follows: voltage, $120 \mathrm{kV}$; electric current, automatic; collimation, 0.5 $\mathrm{mm}$; image reconstruction thickness, $5 \mathrm{~mm}$; and helical pitch, 53 .

Imaging analysis. For both the AH and ND groups, two radiologists (A.N. and Y.U.), who were blinded to the patients' information and laboratory markers, evaluated the patency of the hepatic artery, portal vein, and hepatic vein on the portal venous phase abdominal-to-pelvic $\mathrm{CT}$ in a consensus fashion. The CT perfusion imaging was then analyzed on a workstation (Toshiba Medical Systems). Firstly, the raw volume scan data were processed using software (Body Registration; Toshiba Medical Systems) in order to remove the influence of breathing motion artifacts. Secondly, the registered perfusion data were analyzed using Body Perfusion software based on the dual-input maximum-slope method (11). The parameters generated by the software included the arterial blood flow (AF; $\mathrm{ml} / \mathrm{min} / 100 \mathrm{ml}$ tissue), the portal blood flow (PF; $\mathrm{ml} / \mathrm{min} / 100 \mathrm{ml}$ tissue), and the perfusion index $(\mathrm{PI}, \%)[\mathrm{PI}=\mathrm{AF} /(\mathrm{AF}+\mathrm{PF}) \times 100]$. To obtain these parameters, regions of interest (ROIs) were placed on the abdominal aorta at the level of the celiac axis, the main portal vein, the liver, and the spleen.

The time-density curves of the spleen were used to separate the blood flow in the arterial phase and portal phase, and the maximal slope of the liver time-density curve in each phase was used to calculate both the arterial and portal perfusion. Consequently, a three-dimensional color map (functional map) was produced. A single slice showing the umbilical portion of the portal vein was selected. In a consensus fashion, the same two radiologists (A.N. and Y.U.) measured the perfusion parameters of the right and left liver lobes by placing an ROI on each map. They placed as large a freehand ROI as possible surrounding the edge of each lobe and avoiding large blood vessels. Each perfusion parameter was recorded for the subsequent data analysis.

Statistical analyses. The mean AF, PF, and PI values for each lobe were compared between the AH and ND groups by an independent $t$-test. Perfusion parameters showing a significant difference in this test were used to investigate the relationship between the hemodynamic alterations and the patients' recorded clinical factors. For all patients of both groups, PT, PT-INR, platelet count, ALT, $\mathrm{LDH}$ and ALT/LDH were compared with each perfusion parameter. For the patients of the $\mathrm{AH}$ group, additionally, fibrinogen/fibrin degradation products, D-dimer and ferritin were compared with each perfusion parameter. A linear regression analysis was performed. $p$ Values of less than 0.05 were considered significant.

\section{Results}

For all patients, the patency of the hepatic artery, portal vein, and hepatic vein was confirmed on the portal venous phase abdominal-to-pelvic CT. 
Nishie et al: CT Perfusion in Acute Hepatitis

Table I. Computed tomography perfusion parameters of the acute hepatitis (AH) and no-disease (ND) groups of patients. Data are the mean \pm standard deviation.

\begin{tabular}{llccc}
\hline CT parameter & Lobe & AH group $(\mathrm{n}=14)$ & ND group $(\mathrm{n}=9)$ & $p$-Value \\
\hline $\mathrm{AF}, \mathrm{ml} / \mathrm{min} / 100 \mathrm{ml}$ tissue & Right & $54.0 \pm 24.2$ & $34.6 \pm 17.4$ & 0.05 \\
& Left & $63.4 \pm 29.7$ & $44.7 \pm 16.3$ & 0.10 \\
$\mathrm{PF}, \mathrm{ml} / \mathrm{min} / 100 \mathrm{ml}$ tissue & Right & $121.9 \pm 23.1$ & $138.5 \pm 33.2$ & 0.17 \\
$\mathrm{PI}, \%$ & Left & $104.9 \pm 30.6$ & $131.7 \pm 45.6$ & 0.10 \\
& Right & $30.5 \pm 10.0$ & $20.8 \pm 9.7$ & 0.031 \\
& Left & $38.7 \pm 15.9$ & $28.6 \pm 13.5$ & 0.13 \\
\hline
\end{tabular}

AF: Arterial blood flow; PF: portal blood flow; PI: perfusion index [=AF/(AF+PF)×100].

Table II. Summary of clinical factors recorded for the acute hepatitis $(A H)$ and no-disease $(N D)$ groups. Data are the median (range).

\begin{tabular}{lcc}
\hline Clinical factor & AH group & ND group \\
\hline PT, $\%$ & $40(10-83)$ & $91(83-103)$ \\
PT-INR, $\%$ & $1.81(1.11-5.97)$ & $1.06(0.99-1.11)$ \\
Platelets, $\times 10^{4} / \mu 1$ & $8.5(4.1-20.1)$ & $28.6(18.7-47.3)$ \\
ALT, U/1 & $2,223(20-9,626)$ & $14(7-32)$ \\
LDH, U/1 & $707(186-30,499)$ & $175(137-244)$ \\
ALT/LDH & $1.64(0.07-4.83)$ & $0.09(0.04-0.17)$ \\
FDP, $\mu \mathrm{g} / \mathrm{ml}$ & $22.0(2.7-99.5)$ & - \\
D-Dimers, $\mu \mathrm{g} / \mathrm{ml}$ & $9.4(0.7-48.9)$ & - \\
Ferritin, $\mathrm{ng} / \mathrm{ml}$ & $5,868(162-146,514)$ & - \\
\hline
\end{tabular}

ALT: Alanine aminotransferase; FDP: fibrinogen/fibrin degradation products; LDH: lactate dehydrogenase; PT: prothrombin time; PT-INR: PT-international normalized ratio.

The results of the comparison of the AH and ND groups are summarized in Table I. The mean PI of the right lobe in the $\mathrm{AH}$ group $(30.5 \pm 10.0 \%)$ was significantly higher than that in the ND group $(20.8 \pm 9.7 \%)(p=0.031)$. The mean AF of the right lobe in the AH group ( $54.0 \pm 24.2 \mathrm{ml} / \mathrm{min} / 100 \mathrm{ml}$ tissue) tended to be higher than that in the ND group $(34.6 \pm 17.4 \mathrm{ml} / \mathrm{min} / 100$ $\mathrm{ml}$ tissue) $(p=0.05)$. Although the mean PF of the right lobe in the AH group was slightly higher than that in the ND group, there was no significant difference between the groups.

The clinical factors of the $\mathrm{AH}$ and ND groups are summarized in Table II. The results of the comparison between the PI of the right lobe and clinical factors for all patients of the AH and ND groups and in only patients of the AH group are provided in Table III. For all patients of the $\mathrm{AH}$ and ND groups, the PI of the right lobe increased as the PT decreased $(\mathrm{R}=-0.56, p=0.006)$ and as the PT-INR increased $(\mathrm{R}=0.48, p=0.02$ ) (Figure $1 \mathrm{~A}$ and $\mathrm{B})$. Considering only patients of the AH group, the PI of the right lobe tended also to increase as the PT decreased $(\mathrm{R}=-0.48, p=0.086)$ and as the ALT/LDH ratio decreased $(\mathrm{R}=-0.47, p=0.088)$ (Figure $1 \mathrm{C}$ and D). Representative cases are shown in Figures 2-4.
Table III. Correlation coefficients $(R)$ between the perfusion index of the right liver lobe and clinical factors in patients overall and in the group with acute hepatitis $(\mathrm{AH})$.

\begin{tabular}{lccccc}
\hline \multirow{2}{*}{ Clinical factor } & \multicolumn{2}{c}{ Whole cohort } & & \multicolumn{2}{c}{ AH group } \\
\cline { 2 - 3 } \cline { 6 - 7 } & $\mathrm{R}$ & $p$-Value & & $\mathrm{R}$ & $p$-Value \\
\hline PT, \% & -0.56 & 0.006 & & -0.48 & 0.086 \\
PT-INR, \% & 0.48 & 0.02 & & 0.40 & 0.15 \\
Platelets, $\times 10^{4} / \mu 1$ & -0.11 & 0.62 & & 0.37 & 0.20 \\
ALT, U/1 & 0.28 & 0.19 & & 0.05 & 0.86 \\
LDH, U/1 & 0.32 & 0.14 & & 0.29 & 0.32 \\
ALT/LDH & 0.01 & 0.97 & & -0.47 & 0.088 \\
FDP, $\mu \mathrm{g} / \mathrm{ml}$ & - & - & & 0.33 & 0.24 \\
D-Dimers, $\mu \mathrm{g} / \mathrm{ml}$ & - & - & & 0.38 & 0.19 \\
Ferritin, $\mathrm{ng} / \mathrm{ml}$ & - & - & & 0.30 & 0.29 \\
\hline
\end{tabular}

ALT: Alanine aminotransferase; FDP: fibrinogen/fibrin degradation products; LDH: lactate dehydrogenase; PT: prothrombin time; PT-INR: PT-international normalized ratio.

\section{Discussion}

The main findings of this study are as follows. The mean PI of the right lobe in patients with $\mathrm{AH}$ was significantly higher than that of the patients without liver disease $(p=0.031)$. In addition, the mean AF of the right lobe in the patients with $\mathrm{AH}$ tended to be higher than that of the ND group $(p=0.05)$. In an earlier study using the same CT scanner and analysis method (8), the mean AF and PI values of the right lobe in healthy subjects were $23.2-24.1 \mathrm{ml} / \mathrm{min} / 100 \mathrm{ml}$ tissue and 18.6-19.6\%, respectively, similar to the values measured in the present ND group. Because large branches of the hepatic artery, portal vein, and hepatic vein were patent visually, these results may indicate the presence of a microcirculation disorder in $\mathrm{AH}$.

Hillenbrand et al. reported that there was fibrin in sinusoids, portal vein radicles, and hepatic vein radicles in acute hepatic failure (4). A decrease in the blood flow of 

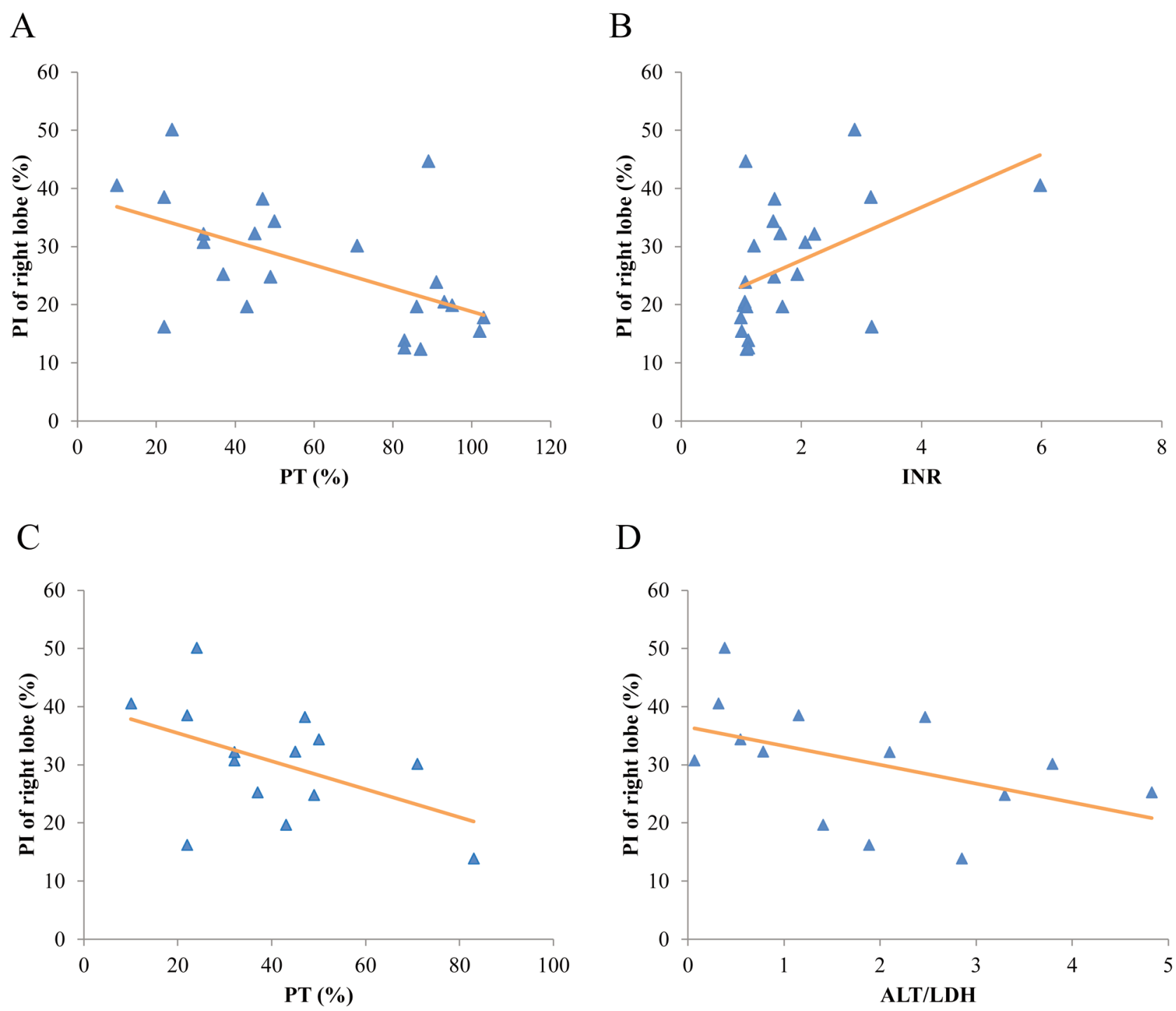

$\mathrm{D}$

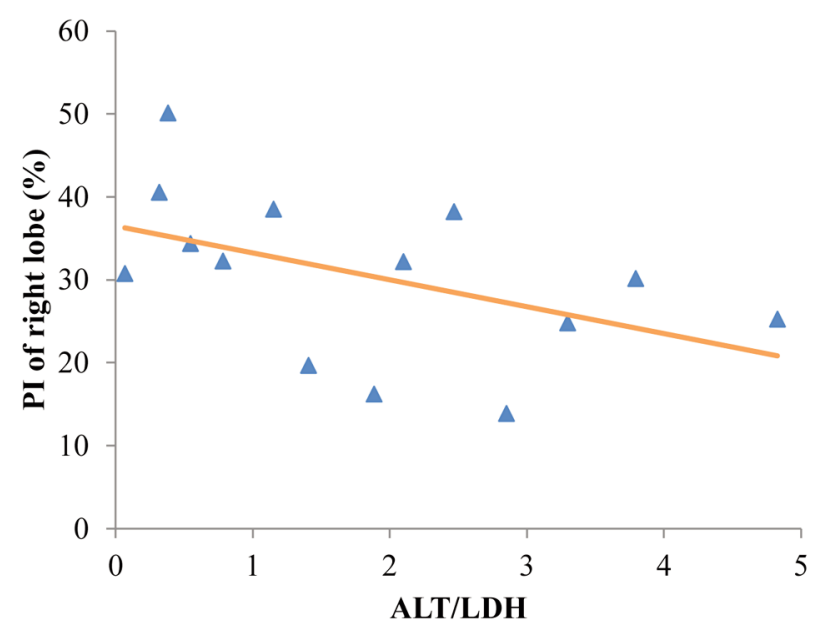

Figure 1. A: The correlation between the prothrombin time (PT) and the perfusion index of the right liver lobe (PI) for the whole patient cohort [acute hepatitis $(A H)$ and no-disease (ND) groups]. The correlation coefficient was $-0.56 . P I(\%)=-0.20 \times P T(\%)+38.9$. B: The correlation between PT-international normalized ratio (INR) and PI for the whole patient cohort. The correlation coefficient was 0.48. PI $(\%)=4.55 \times$ PT-INR+18.6. $C$ : The correlation between PT and PI of the right liver lobe for patients of the AH group. The correlation coefficient was $-0.48 . P I(\%)=-0.24 \times$ $P T(\%)+40.3$. D: The correlation between alanine aminotransferase/lactate dehydrogenase $(A L T / L D H)$ ratio and the PI patients of the AH group. The correlation coefficient was -0.47 . PI $(\%)=-3.24 \times A L T / L D H+36.5$.

sinusoids and the hepatic vein leads to a decrease in portal perfusion (12). The mean PF of the right lobe in the $\mathrm{AH}$ group of the present study was slightly lower than that of the ND group, although the difference was not significant. We thus hypothesize that arterial perfusion may increase in association with decrease in portal perfusion. To our knowledge, the present study is the first report to directly reveal hemodynamic alteration in $\mathrm{AH}$.

Kanematsu et al. reported early patchy enhancement of the liver on the arterial phase of dynamic magnetic resonance imaging in $\mathrm{AH}$ (13). Although they suggested this was due to a leakage of contrast material to increased extracellular space due to hepatocyte necrosis and capillary vessel injury, we speculate that their finding may also represent a heterogeneous increase in arterial perfusion. In the present series, six patients recovered after arterial steroid injection therapy for the liver. Corticosteroid suppresses the function of activated macrophages (5). Although there was no significant correlation between the PI of the right lobe and the level of ferritin, which is thought to be produced by 

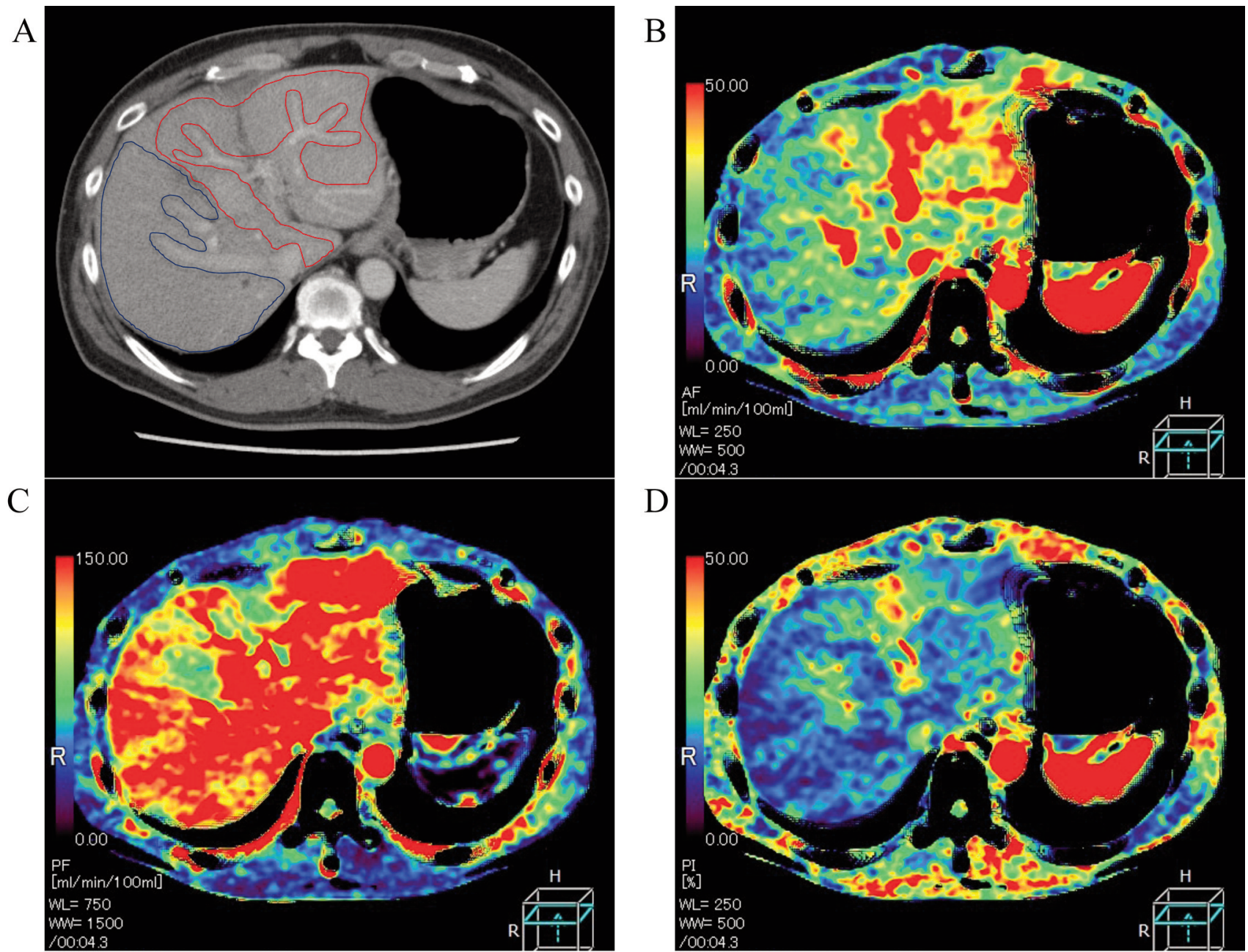

Figure 2. A 55-year-old man underwent computed tomography perfusion imaging for the evaluation of gastric cancer. He had no underlying liver disease, liver dysfunction, or liver metastasis. A: Portal venous phase image: No abnormality in morphology or attenuation was observed in the liver. Regions of interests placed on the right (blue) and left (red) lobes on each map are shown. B: Map of the arterial blood flow (AF): The AF values of the right and left lobes were $26.4 \mathrm{ml} / \mathrm{min} / 100 \mathrm{ml}$ tissue and $40.4 \mathrm{ml} / \mathrm{min} / 100 \mathrm{ml}$ tissue, respectively. C: Map of the portal blood flow (PF): The PF values of the right and left lobes were $127.4 \mathrm{ml} / \mathrm{min} / 100 \mathrm{ml}$ tissue and $139.8 \mathrm{ml} / \mathrm{min} / 100 \mathrm{ml}$ tissue, respectively. D: Perfusion index (PI) map: The PI values of the right and left lobes were $17.8 \%$ and $24.1 \%$, respectively.

activated macrophages (14), hypercytokinemia originating from such macrophages might cause a microcirculation disorder in the liver.

We observed that the PI of the right lobe increased as the PT decreased and as the PT-INR increased for patients overall, and that the PI of the right lobe tended to increase as the PT and ALT/LDH ratio decreased for patients of the AH group. PT and PT-INR are correlated with the degree of liver failure. $\mathrm{LDH}$ production is elevated in hypoxic conditions (15). The ALT/LDH ratio may therefore represent hepatic injury or necrosis derived from a microcirculation disorder. The reason why the PI of the right lobe was not correlated with the ALT/LDH ratio for all patients of the $\mathrm{AH}$ and ND groups might be as follows. The ALT/LDH ratio was basically low in the ND group. Contrarily, although this ratio tended to be higher in the $\mathrm{AH}$ group, it can decrease when hypoxia is severe. These results suggest that the PI of the right liver lobe may be associated with the severity of acute hepatic failure. Although several parameters predictive of acute hepatic failure have been proposed, including coagulopathy, jaundice, etiology, and aging (1, 16-19), a reliable and widely accepted procedure for distinguishing self-limiting cases from fatal ones has not been established $(19,20)$. The PI of the right lobe may be a predictive parameter in $\mathrm{AH}$ for use in the prediction of therapeutic effects and prognosis. 

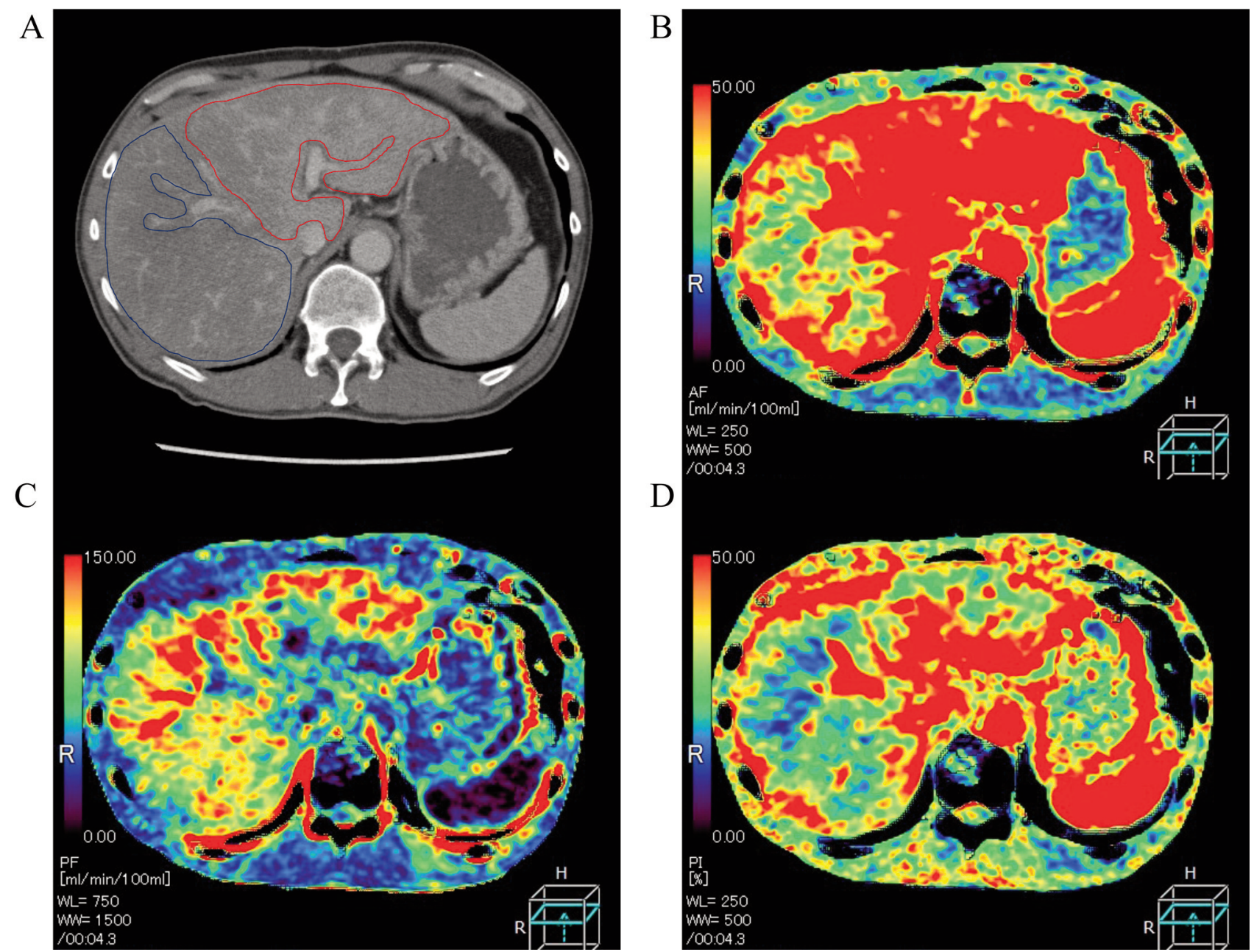

Figure 3. A 55-year-old man underwent computed tomography perfusion imaging for the evaluation of acute hepatitis. He had no underling liver disease, and drug induction was suspected as the cause of acute hepatic failure. The prothrombin time (PT) and alanine aminotransferase/lactate dehydrogenase $(A L T / L D H)$ ratio were $10 \mathrm{~ms}$ and 0.32 , respectively. A: Portal venous phase image: No abnormality in morphology, but slightly heterogeneous attenuation was observed in the liver. Regions of interest placed on the right (blue) and left (red) lobes on each map are indicated. B: Map of the arterial blood flow (AF): The AF values of the right and left lobes were $53 \mathrm{ml} / \mathrm{min} / 100 \mathrm{ml}$ tissue and $84.8 \mathrm{ml} / \mathrm{min} / 100 \mathrm{ml}$ tissue, respectively. C: Map of the portal blood flow (PF): The PF values of the right and left lobes were $79.8 \mathrm{ml} / \mathrm{min} / 100 \mathrm{ml}$ tissue and $56.2 \mathrm{ml} / \mathrm{min} / 100$ ml tissue, respectively. D: Perfusion index (PI) map: The PI values of the right and left lobes were $40.6 \%$ and $60.6 \%$, respectively. The patient was treated with plasma exchange and arterial steroid injection therapy for the liver. This case had a high PI, a low PT, and a low ALT/LDH.

The AF and PF in the liver vary in absolute value. For example, Motosugi et al. reported that the $\mathrm{AF}$ and $\mathrm{PF}$ values of the right lobe ranged from 20 to $57 \mathrm{ml} / \mathrm{min} / 100 \mathrm{ml}$ and from 24 to $253 \mathrm{ml} / \mathrm{min} / 100 \mathrm{ml}$, respectively (9). It thus seems reasonable to use the PI, which is a relative value, as an indicator of hemodynamic alterations in the liver.

We analyzed hepatic perfusion in the right and left lobes separately because there was a slight difference in perfusion between the lobes in previous reports (7-9). For example, the mean PF of the left lobe in healthy individuals was 95.6-131.4 $\mathrm{ml} / \mathrm{min} / 100 \mathrm{ml}$ tissue, and that of the right lobe was 114.0 -
$118.7 \mathrm{ml} / \mathrm{min} / 100 \mathrm{ml}$ tissue (8). There was also a difference in perfusion among the four segments of the left lobe compared to the right lobe. It is not yet known why the values of the right lobe reflect microcirculation disorder better than those of the left lobe, and we considered the following plausible reasons. (i) Artifacts derived from the stomach (e.g., air or motion) may affect the measurement of perfusion in the left lobe. (ii) The blood perfusion of the left lobe may be affected by gravity. (iii) Small areas outside of the liver may be included when placing the ROI on the left lobe on perfusion maps. Measurements on the right lobe thus seem preferable for investigation. 

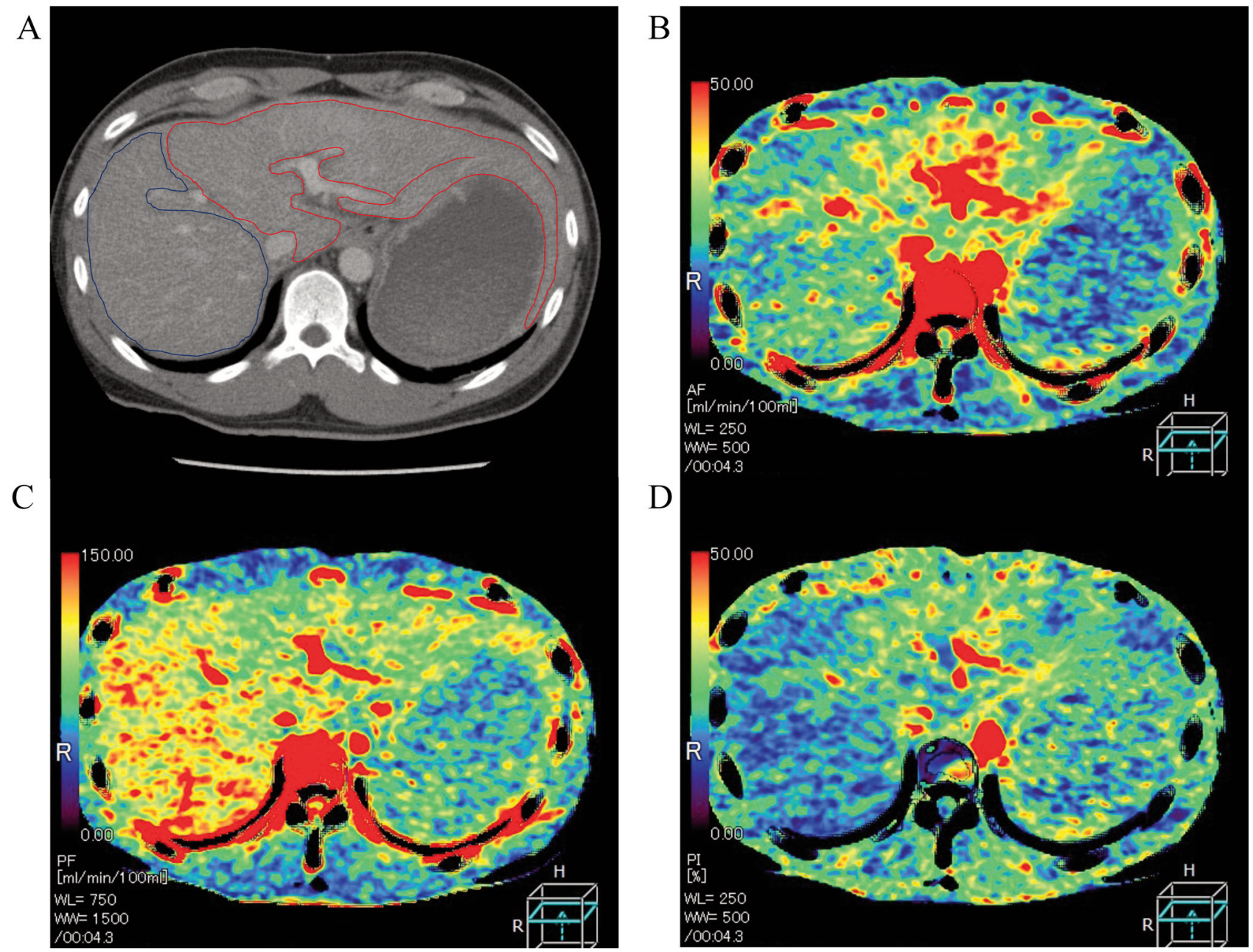

Figure 4. A 39-year-old man underwent CT perfusion imaging for the evaluation of acute hepatitis. He was a carrier of hepatitis virus B, which was thought to be the cause of his acute hepatic failure. The prothrombin time (PT) and alanine aminotransferase/lactate dehydrogenase (ALT/LDH) ratio were $37 \mathrm{~ms}$ and 4.8, respectively. A: Portal venous phase image: No abnormality in morphology was observed in the liver. Regions of interest placed on the right (blue) and left (red) lobes on each map are indicated. B: Map of the arterial blood flow (AF): The AF values of the right and left lobes were $32.6 \mathrm{ml} / \mathrm{min} / 100 \mathrm{ml}$ tissue and $38.2 \mathrm{ml} / \mathrm{min} / 100 \mathrm{ml}$ tissue, respectively. C: Map of the portal blood flow (PF): The PF values of the right and left lobes were $99.5 \mathrm{ml} / \mathrm{min} / 100 \mathrm{ml}$ tissue and $105.5 \mathrm{ml} / \mathrm{min} / 100 \mathrm{ml}$ tissue, respectively. D: Perfusion index (PI) map: The PI values of the right and left lobes were $25.3 \%$ and $30.1 \%$, respectively. He was treated with plasma exchange and arterial steroid injection therapy for the liver. This case had a low PI, a relatively low PT, and a high ALT/LDH ratio.

The effective dose of our CT perfusion imaging was 15.5 $\mathrm{mSv}$. At our Institution, the effective dose of three-phase abdominal CT using 64 multi-detector-row CT was 28.9 $\mathrm{mSv}$. According to our approximation, the total effective dose of the CT perfusion imaging and portal venous phase abdominal-to-pelvic CT was lower than that of the threephase abdominal CT. Therefore, CT perfusion imaging can be clinically justified.

There are several limitations to this study. Firstly, the number of patients enrolled was relatively small. Generally, few patients with severe acute liver failure are transferred to our Institution, and it was therefore difficult to collect a number of such cases. Secondly, the patients' underlying liver diseases causing $\mathrm{AH}$ varied. Thirdly, the ND group (healthy population) comprised patients with gastric cancer; however, none had underlying liver disease, liver dysfunction, or liver metastasis. Fourthly, a tissue biopsy of the liver was available for only two patients. No radiological-pathological comparison was possible. Fifthly, it was difficult to avoid small vessels when placing the ROI on the map. Finally, none of the patients underwent follow-up CT perfusion after treatment. The recovery of CT perfusion parameters might further support our hypothesis. 
In conclusion, the PI of the right liver lobe in patients with acute hepatitis was higher than that in the population without liver disease, and this index might be a useful parameter predictive for the severity of acute hepatic failure.

\section{Conflicts of Interest}

Akihiro Nishie was a staff member of joint research departments in Kyushu University with Canon Medical Systems Corporation. He received a research grant from Canon Medical Systems Corporation. The other Authors have no conflicts of interest.

\section{Authors' Contributions}

Akihiro Nishie: Conceptualization, methodology, writing - original draft. Yasuhiro Ushijima: Methodology, data curation, writing original draft. Yukihisa Takayama: Formal analysis, writing review and editing. Nobuhiro Fujita: Formal analysis, writing review and editing. Yuichiro Kubo, Keisuke Ishimatsu, Daisuke Tsurumaru: Investigation, writing - review and editing. Motoyuki Kohjima: Resources, writing - review and editing. Kousei Ishigami: Writing - review and editing, supervision, project administration.

\section{Acknowledgements}

The Authors thank Professor Yoshihiro Ogawa, Department of Medicine and Bioregulatory Science, Kyushu University, for providing the clinical information for this article. This study was supported by Canon Medical Systems Corporation.

\section{References}

1 Takikawa Y, Endo R, Suzuki K, Fujiwara K, Omata M and Fulminant Hepatitis Study Group of Japan: Prediction of hepatic encephalopathy development in patients with severe acute hepatitis. Dig Dis Sci 51(2): 359-364, 2006. PMID: 16534681. DOI: $10.1007 / \mathrm{s} 10620-006-3138-7$

2 Chun LJ, Tong MJ, Busuttil RW and Hiatt JR: Acetaminophen hepatotoxicity and acute liver failure. J Clin Gastroenterol 43(4): 342-349, 2009. PMID: 19169150. DOI: 10.1097/MCG. 0b013e31818a3854

3 Wu Z, Han M, Chen T, Yan W and Ning Q: Acute liver failure: mechanisms of immune-mediated liver injury. Liver Int 30(6): 782-794, 2010. PMID: 20492514. DOI: 10.1111/j.1478-3231. 2010.02262.x

4 Hillenbrand P, Parbhoo SP, Jedrychowski A and Sherlock S: Significance of intravascular coagulation and fibrinolysis in acute hepatic failure. Gut 15(2): 83-88, 1974. PMID: 4820641. DOI: 10.1136/gut.15.2.83

5 Possamai LA, Thursz MR, Wendon JA and Antoniades CG: Modulation of monocyte/macrophage function: a therapeutic strategy in the treatment of acute liver failure. J Hepatol 61(2): 439445, 2014. PMID: 24703954. DOI: 10.1016/j.jhep.2014.03.031

6 Zhuang ZG, Qian LJ, Wang BX, Zhou Y, Li QG, Xu JR and Cheng YF: Computed tomography perfusion in living donor liver transplantation: an initial study of normal hemodynamic changes in liver grafts. Clin Transplant 23(5): 692-699, 2009. PMID: 19473203. DOI: 10.1111/j.1399-0012.2009.00991.x
7 Kanda T, Yoshikawa T, Ohno Y, Kanata N, Koyama H, Takenaka $\mathrm{D}$ and Sugimura K: CT hepatic perfusion measurement: comparison of three analytic methods. Eur J Radiol 81(9): 20752079, 2012. PMID: 21802233. DOI: 10.1016/j.ejrad.2011.07.003

8 Kanda T, Yoshikawa T, Ohno Y, Fujisawa Y, Kanata N, Yamaguchi M, Seo Y, Yano Y, Koyama H, Kitajima K, Takenaka $\mathrm{D}$ and Sugimura K: Perfusion measurement of the whole upper abdomen of patients with and without liver diseases: initial experience with 320-detector row CT. Eur J Radiol 81(10): 2470-2475, 2012. PMID: 22055684. DOI: 10.1016/j.ejrad. 2011.10.009

9 Motosugi U, Ichikawa T, Sou H, Morisaka H, Sano K and Araki $\mathrm{T}$ : Multi-organ perfusion CT in the abdomen using a 320 detector row CT scanner: preliminary results of perfusion changes in the liver, spleen, and pancreas of cirrhotic patients. Eur J Radiol 81(10): 2533-2537, 2012. PMID: 22227262. DOI: 10.1016/j.ejrad.2011.11.054

$10 \mathrm{Wm} \mathrm{T}, \mathrm{L} \mathrm{S}, \mathrm{C} \mathrm{K}, \mathrm{K} \mathrm{E}, \mathrm{T} \mathrm{H}, \mathrm{H} \mathrm{B}, \mathrm{T} \mathrm{K}, \mathrm{K} \mathrm{N}, \mathrm{M} \mathrm{H}$ and S K: Quantification of hemodynamic changes in chronic liver disease: Correlation of perfusion-CT data with histopathologic staging of fibrosis. Acad Radiol 26(9): 1174-1180, 2019. PMID: 30528750. DOI: $10.1016 /$ j.acra.2018.11.009

11 Miles KA, Hayball M and Dixon AK: Colour perfusion imaging: a new application of computed tomography. Lancet 337(8742): 643-645, 1991. PMID: 1671994. DOI: 10.1016/0140-6736(91) 92455-b

12 Nishie A, Yoshimitsu K, Irie H, Tajima T, Hirakawa M, Ishigami K, Ushijima Y, Okamoto D, Nishihara Y, Taketomi A and Honda $\mathrm{H}$ : Radiological detectability of minute hepatic venous invasion in hepatocellular carcinoma. Eur J Radiol 70(3): 517-524, 2009. PMID: 18406555. DOI: 10.1016/j.ejrad.2008.02.021

13 Kanematsu M, Danet MI, Leonardou P, Mastropasqua M, Mosetti MA, Braga L, Woosley JT and Semelka RC: Early heterogeneous enhancement of the liver: magnetic resonance imaging findings and clinical significance. J Magn Reson Imaging 20(2): 242-249, 2004. PMID: 15269949. DOI: 10.1002/jmri.20097

14 Allen CE, Yu X, Kozinetz CA and McClain KL: Highly elevated ferritin levels and the diagnosis of hemophagocytic lymphohistiocytosis. Pediatr Blood Cancer 50(6): 1227-1235, 2008. PMID: 18085676. DOI: 10.1002/pbc.21423

15 Rees BB, Bowman JA and Schulte PM: Structure and sequence conservation of a putative hypoxia response element in the lactate dehydrogenase-B gene of Fundulus. Biol Bull 200(3): 247-251, 2001. PMID: 11441966. DOI: 10.2307/1543505

16 Miyake Y, Sakaguchi K, Iwasaki Y, Ikeda H, Makino Y, Kobashi $\mathrm{H}$, Araki Y, Ando M, Kita K and Shiratori Y: New prognostic scoring model for liver transplantation in patients with nonacetaminophen-related fulminant hepatic failure. Transplantation 80(7): 930-936, 2005. PMID: 16249741. DOI: 10.1097/01.tp.000 0173651.39645 .35

17 Dhiman RK, Seth AK, Jain S, Chawla YK and Dilawari JB: Prognostic evaluation of early indicators in fulminant hepatic failure by multivariate analysis. Dig Dis Sci 43(6): 1311-1316, 1998. PMID: 9635624. DOI: 10.1023/a:1018876328561

18 Elinav E, Ben-Dov I, Hai-Am E, Ackerman Z and Ofran Y: The predictive value of admission and follow up factor V and VII levels in patients with acute hepatitis and coagulopathy. J Hepatol 42(1): 82-86, 2005. PMID: 15629511. DOI: 10.1016/ j.jhep.2004.09.009 
19 Kotoh K, Enjoji M, Nakamuta M, Yoshimoto T, Kohjima M, Morizono S, Yamashita S, Horikawa Y, Yoshimitsu K, Tajima T, Asayama Y, Ishigami K and Hirakawa M: Arterial steroid injection therapy can inhibit the progression of severe acute hepatic failure toward fulminant liver failure. World J Gastroenterol 12(41): 6678-6682, 2006. PMID: 17075983. DOI: 10.3748/wjg.v12.i41.6678

20 Zheng YX, Zhong X, Li YJ and Fan XG: Performance of scoring systems to predict mortality of patients with acute-on- chronic liver failure: A systematic review and meta-analysis. J Gastroenterol Hepatol 32(10): 1668-1678, 2017. PMID: 28303605. DOI: $10.1111 /$ jgh.13786

Received September 12, 2021

Revised September 29, 2021

Accepted September 30, 2021 